\title{
Near-infrared digital photography to estimate snow correlation length for microwave emission modeling
}

\author{
Ally Mounirou Toure, ${ }^{1, *}$ Kalifa Goïta, ${ }^{1}$ Alain Royer, ${ }^{1}$ Christian Mätzler, ${ }^{2}$ \\ and Martin Schneebeli ${ }^{3}$ \\ ${ }^{1}$ CARTEL, Département de Géomatique Appliquée, Université de Sherbrooke, \\ 2500 Blvd Université, Sherbrooke, Québec J1K 2R1, Canada \\ ${ }^{2}$ Institute of Applied Physics (IAP), University of Bern, Sidlerstrasse 5, 3012 Bern, Switzerland \\ ${ }^{3}$ WSL Institute for Snow and Avalanche Research (SLF), Flüelastrasse 11, 7260 Davos-Dorf, Switzerland \\ *Corresponding author: ally.toure@usherbrooke.ca
}

Received 9 May 2008; revised 3 November 2008; accepted 22 October 2008;

posted 14 November 2008 (Doc. ID 95774); published 12 December 2008

\begin{abstract}
The study is based on experimental work conducted in alpine snow. We made microwave radiometric and near-infrared reflectance measurements of snow slabs under different experimental conditions. We used an empirical relation to link near-infrared reflectance of snow to the specific surface area (SSA), and converted the SSA into the correlation length. From the measurements of snow radiances at 21 and $35 \mathrm{GHz}$, we derived the microwave scattering coefficient by inverting two coupled radiative transfer models (the sandwich and six-flux model). The correlation lengths found are in the same range as those determined in the literature using cold laboratory work. The technique shows great potential in the determination of the snow correlation length under field conditions. ( 2008 Optical Society of America OCIS codes: $\quad 280.0280,280.4991,120.5630,120.5700$.
\end{abstract}

\section{Introduction}

There are various radiative transfer models designed to simulate the snow emission in the microwave range, e.g., the Helsinki University of Technology snow emission model (HUT, [1]), the microwave emission model of layered snowpacks (MEMLS, [2]), the dense medium radiative transfer model (DMRT, [3]), and the strong fluctuation theory (SFT, $[4,5])$. HUT and DMRT on the one hand use the snow grain size as a structure parameter; on the other hand, MEMLS and SFT use the correlation length to quantify the snow microwave scattering.

For practical purposes, snow hydrologists have defined snow grain size as the greatest diameter of

0003-6935/08/366723-11\$15.00/0

(C) 2008 Optical Society of America prevailing grains in the snow layer [묵]. However, it has been shown that using the maximum extent of characteristic grains can lead to a strong overestimation of the effective grain size applicable in scattering models [8]. This optical grain size corresponds to the diameter of noncontacting spheres with the same surface area and the same ice volume as the snowpack under consideration, and thus with the same specific surface area (SSA) [9]. For example, the effective size of long needles is quite small, because the value is close to the diameter of the needles; for disks of large diameter, the optical grain size could also be small, depending on their thickness [10]. Stellar snow crystals can have a maximum extent of $1 \mathrm{~cm}$, but their thickness is only 20 to $40 \mu \mathrm{m}$. The optical grain size and the correlation length are the appropriate parameters to describe electromagnetic wave scattering [8]. The correlation length can be understood as $\bar{a}$ measure of the average 
distance beyond which variations of the dielectric constant in one region of space become uncorrelated with those in another region [11]. At a distance greater than the correlation length, the values can be considered random. The correlation length $p_{c}$ is proportional to the optical grain size $D_{o}$, and both are inversely proportional to SSA [12].

Also in the computation of the microwave scattering of a granular medium such as snow, the physically meaningful structure parameters are $p_{c}$ and $D_{o}[\underline{8}, \underline{10}, \underline{13}, \underline{14}]$. One way to determine these quantities is through the measurement of SSA. Until recently, there was no easy way for a practical determination of the SSA under field conditions. Because of that, there have been some attempts to establish empirical relationships between the correlation length and the snow grain size. The approaches are approximations based on observations made at one site. The empirical formulas cannot be used in all circumstances. The concept of using the scattering of light to determine SSA and thus $D_{o}$ was first speculated by Giddings and Lachapelle [15] and Warren and Wiscombe [16] found first confirmation by using the Mie theory to describe the optical properties of snow in the solar spectrum. The dependence of snow reflectance on grain size was used in optical remote sensing to map grain size in the surface snow layer $[17,18]$. The fact that SSA can be directly converted to optical grain size was shown by Mitchell [19]. The traditional method of determining SSA involves cold-laboratory work, which is time consuming and laborious $[20,21]$. Therefore, an important model-input parameter has so far been unavailable in the field.

Advances in digital camera technology have made it possible to measure the snow reflectance using a simple digital camera fitted with an appropriate filter. An exponential relationship was found in [22] between the SSA and the reflectance of snow at the near-infrared (NIR) spectrum. Matzl and Schneebeli used the relationship to determine the SSA of snow profile with an uncertainty of less than 15\%. A coupled contact illumination probe and field spectroradiometer was used by Painter et al. [23] to determine the optical grain size under field conditions with greater precision than the NIR technique. But the NIR technique has much higher spatial resolution imagery of SSA (a $1 \mathrm{~mm}$ thick layer can be clearly identified) than the spectrometer measurements technique ( $2 \mathrm{~cm}$ spatial resolution). This characteristic makes the NIR method interesting because it can also be used to accurately determine the snow stratigraphy, and locate ice lenses, hard crust, and thin layers in the profile [22]. All these parameters greatly influence the snow microwave emission. Furthermore, NIR photography is simpler and more convenient for field work. The equipment is basic; only a digital camera and the Spectralon for calibration are needed. No source of artificial light is needed.
In this study, we considered the NIR technique for determination of the snow SSA and its correlation length. To check whether the NIR reflectance can be used to determine the correlation length for use in snow microwave emission modeling, we carried out experimental work at the snow station of the Swiss Federal Institute for Avalanche Research at Weissfluhjoch, Davos. We performed two types of experiment:

1. The first was to measure the snow slab brightness temperature at 21 and $35 \mathrm{GHz}$ in different experimental conditions, and from that we retrieved the absorption and scattering coefficients through the inversion of the six-flux radiative transfer equations [24].

2 . The second experiment was to determine the SSA of the snow sample using NIR digital photography, and from that information, to compute the correlation length of the samples.

\section{Experiments}

\section{A. Geographical Location of the Site}

The site of the measurements is Weissfluhjoch, Davos, Switzerland, in the Dorftälli, a locally flat area at $2540 \mathrm{~m}$ above sea level. This is the location of the snow station for the Swiss Federal Institute for Snow and Avalanche Research. At this station, regular weather and snow parameter measurements are made. The measurements were conducted from 12 to 22 April 2006, i.e., just before the spring melt began.

\section{B. Material}

We used two portable, linearly polarized radiometers operating at 21 and $35 \mathrm{GHz}$ (Table 1) built at the Institute of Applied Physics, University of Bern. A blackbody box kept at ambient temperature was used to calibrate the radiometers. An aluminum plate was used to serve as the perfect reflector, a $5 \mathrm{~cm}$ thick blackbody plate was used to serve as the perfect absorber of microwave radiations, and a $3 \mathrm{~cm}$ thick styrofoam plate was used to thermally insulate and to help support the slab. The output voltage of the radiometers was recorded by a data logger. The voltage is linearly related to the brightness temperature $(\mathrm{Tb})$. The digital camera used for the NIR photography was a Sony Cybershot DSC$\mathrm{P} 200$. The charge-coupled device (CCD) used in the camera is sensitive to NIR light. A filter was placed

Table 1. Properties of the Portable Dicke Radiometers

\begin{tabular}{lcc}
\hline \multicolumn{1}{c}{ Properties } & Radiometer 1 & Radiometer 2 \\
\hline Center frequency $f[\mathrm{GHz}]$ & 21 & 35 \\
Bandwidth $\Delta f[\mathrm{GHz}]$ & 0.8 & 0.8 \\
Integration time $t[\mathrm{~S}]$ & $1-8$ & $1-8$ \\
Sensibility [K] & 0.1 & 0.1 \\
3 dB beam width $\varphi$ & $9^{\circ}$ & $9^{\circ}$ \\
Horn antenna & Pyramidal & Pyramidal \\
\hline
\end{tabular}


over the CCDs to block the visible light. The wavelength of the detected light is in the range of 840 and $940 \mathrm{~nm}$. The photos were taken under diffuse light with the camera placed on a tripod at approximately $1 \mathrm{~m}$ from the sample. When there was direct radiation, a white curtain was used to create a diffuse light.

\section{Tb Measurement Procedures}

The principal setup of the experiment is shown in Fig. 1. The radiometers were mounted on a frame that allows easy variation of incidence angle and change of polarization. The snow sample is placed on the styrofoam plate on a metal table. Between the snow and the sample, an absorber or metal plate was inserted. The samples were placed near the radiometer antennas to ensure that all the measured radiation came from the sample surface and volume. A box at the rear contains the device for adjusting the voltage of the power supply, as well as a car battery as a spare power supply in case of failure of the central power generator of the snow station. The output voltages of the radiometers were recorded by a datalogger [polycorder (PC)].

a. Removal of the slab. The preparation of the snow samples was one of the most delicate tasks of the measurement procedure. This method requires homogeneous samples of dry snow to study their microwave properties as a function of their structure. To measure the brightness temperature $(\mathrm{Tb})$ of the snow, we first had to mechanically remove $70 \times$ $60 \mathrm{~cm}$ blocks of snow with thicknesses varying between 9 and $20 \mathrm{~cm}$. Snow was excavated at a previously undisturbed site to ensure that the layers were as homogeneous as possible. The snowpack was examined to find layers of sufficient thickness. Once a layer was chosen, a sample was prepared

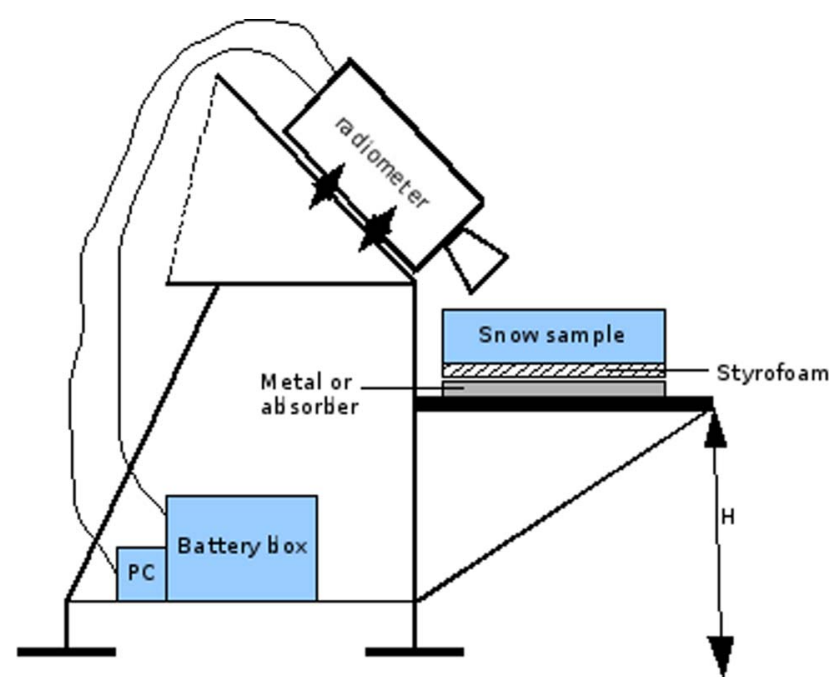

Fig. 1. (Color online) Experimental setup: the radiometer is mounted on a frame directed at the snow sample $(9$ to $20 \mathrm{~cm})$ which is placed on a $3 \mathrm{~cm}$ thick styrofoam plate on a metal table. Between the snow and the sample an absorber or metal plate was inserted. The polycorder (PC) and the battery box are also shown. by cutting the layer with metal plates, taking great care to keep the slab undisturbed. The sample was then placed on a $3 \mathrm{~cm}$ thick styrofoam plate to help support the sample. Tests done by Wiesmann et al. [24] showed that the transmissivity of the styrofoam is better than 0.99 in all conditions for the frequency range used here. The styrofoam had the advantage of thermally insulating the samples from the various underlying bases during handling and measurements. In the case of new snow, the handling was very difficult, even on the styrofoam plate, because those samples were likely to break apart or to show fissures, requiring the preparation of new samples. The surface of the samples was then smoothed by a straight metal edge, a process that had to be performed very accurately. The slabs were then placed on a platform in front of the radiometers for measurements (Fig. 1). Thirty-three samples were used for the experimental measurements. The radiometric measurements were taken immediately after the sample was extracted. After the radiometric measurements were finished, the snow temperature was determined at different points near the center and the edges of the samples. The maximum temperature difference did not exceed $2 \mathrm{~K}$. The snow density was also measured. The density ranged from 69 to $400 \mathrm{~kg} / \mathrm{m}^{3}$.

b. Measurements. The brightness temperatures of the snow slabs were measured in two different situations to determine the reflectivity and the transmissivity of the samples: (a) the snow slab on the aluminum plate at $45^{\circ}$ angle of incidence, at both vertical and horizontal polarization; (b) the snow slab on the absorber at $45^{\circ}$ angle of incidence, at both horizontal and vertical polarization. For calibration purposes, the sky brightness temperature and that of the blackbody box at ambient temperature were also measured. The depth at which the sample was taken was also recorded.

\section{Near-Infrared Photography}

Before taking the NIR photos, the side of the slab was carefully smoothed with a blade. The calibration targets were then placed on the sample (Fig. 2). The targets were manufactured from Spectralon standards with NIR reflectances of $50 \%$ and $99 \%$. The distance between the camera and the profile wall was approximately $1 \mathrm{~m}$. To compensate for tilting or turning of the camera with respect to the surface of the slab, a geometric correction of the digital image was needed. The correction required at least three targets to be inserted on the profile with a known distance from each other and known geometry (Fig. 2). The nearest neighbor method was used to transform the target coordinates in the digital image congruently with their original geometry and distance [22]. The NIR reflectance (nir) of the snow was calibrated with regard to the pixel intensity of the targets:

$$
\operatorname{nir}=a+b i
$$




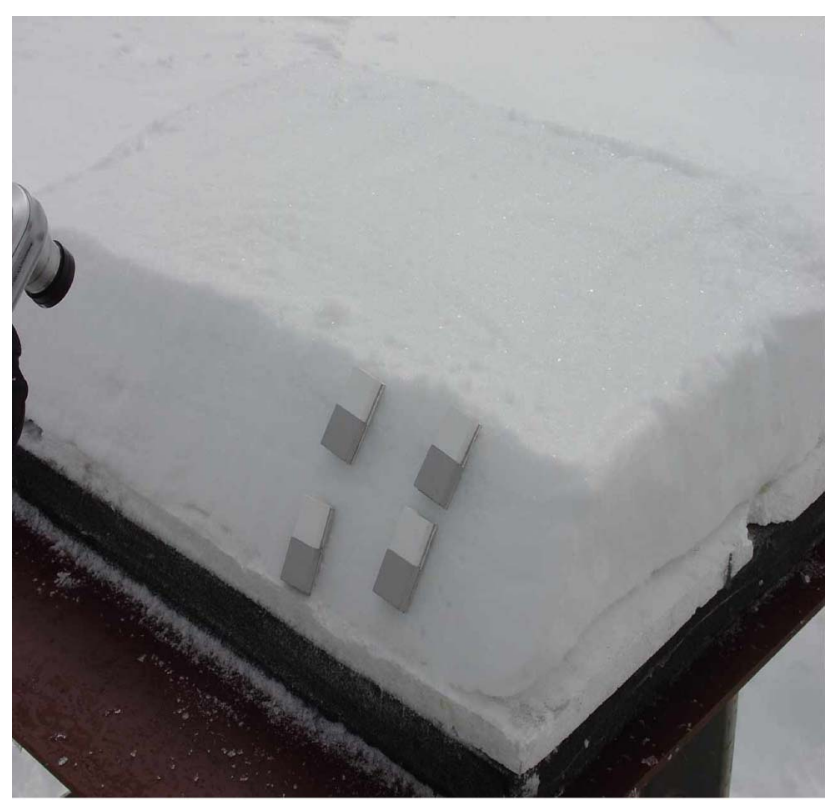

Fig. 2. (Color online) Preparation of the snow slab for the NIR photography. Four gray-white targets are placed on a smoothed snow side.

where $i$ is the intensity of each pixel and $a$ and $b$ are determined by a linear regression on the pixel intensities of gray-scale standards.

\section{E. Correlation of Reflectance and SSA}

It was found by Matzl and Schneebeli [22] that the correlation between the NIR reflectance of the snow samples calculated from the calibrated digital image and their SSA is about $90 \%$. The spatial resolution of the imagery is very high. In images covering between 0.5 and $1 \mathrm{~m}^{2}$, even layers of $1 \mathrm{~mm}$ thickness can be documented and measured (Fig. 3). The error increases with increasing SSA from $\overline{3} \%$ for SSA below $5 \mathrm{~mm}^{-1}$ to about $15 \%$ for SSA values above $25 \mathrm{~mm}^{-1}$.

$$
\mathrm{SSA}=A \cdot e^{\mathrm{nir} / b},
$$

where nir is in $\%, A=0.017 \pm 0.009 \mathrm{~mm}^{-1}$, and $b=$ $12.222 \pm 0.842[22]$.

\section{Radiometric Properties of the Snow Slab}

The aim of the radiometric property studies is to understand how various $\mathrm{Tb}$ are related to the emissivity $(e)$, reflectivity $(r)$ and transmissivity $(t)$ of a snow slab, and eventually to the snow scattering and absorption coefficients.

To connect the measured $\mathrm{Tb}$ to the snow internal scattering and the reflections at the interfaces, we used the simple sandwich model based on the radiative transfer proposed and developed by Wiesmann et al. [24].

We made two types of measurement:

a. Brightness temperature of snow on metal plate $\left(\mathrm{Tb}_{\text {met }}\right)$. Figure $4(\mathrm{a})$ presents the situation of a snow slab with thickness $d$ on a metal plate. The

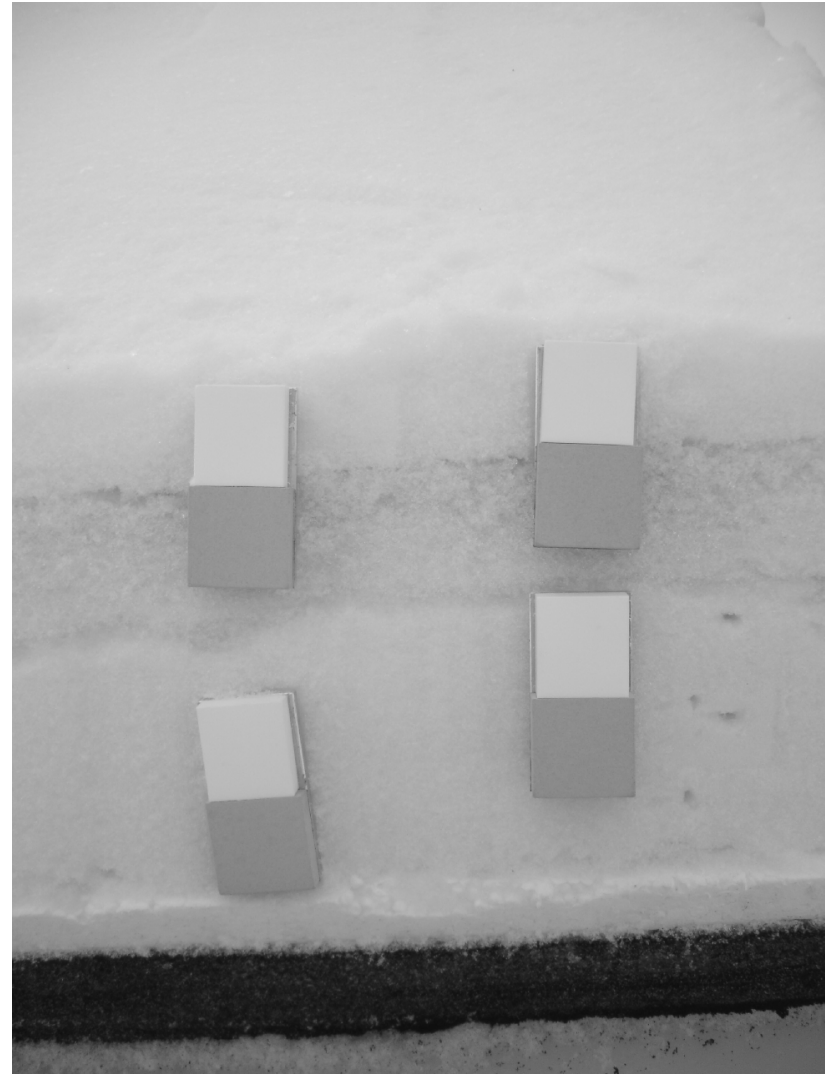

Fig. 3. (Color online) NIR photo of a $13 \mathrm{~cm}$ thick inhomogeneous slab laying on a blackbody and styrofoam slabs: the top layer is newly fallen snow with high NIR (SSA $=38.5 / \mathrm{mm}$ ), the middle layer is refrozen snow with very low NIR $(\mathrm{SSA}=7.7 / \mathrm{mm})$, and the bottom layer consists of rounded snow $(\mathrm{SSA}=17.5 / \mathrm{mm})$.

upwelling brightness temperature $\mathrm{Tb}_{\text {met }}$ is composed of the emitted snow radiation, caused by the physical snow temperature $T_{\text {snow }}$ of the sky radiation $\mathrm{Tb}_{\text {sky }}$ that is reflected from the slab (total reflectivity $r_{\text {met }}$ ), and of $\mathrm{Tb}_{\text {sky }}$ that is transmitted through the snow slab and reflected by the metal plate. The upwelling brightness temperature of the snow on the metal plate $\mathrm{Tb}_{\text {met }}$ is

$$
\mathrm{Tb}_{\mathrm{met}}=\left(1-r_{\mathrm{met}}\right) T_{\mathrm{snow}}+r_{\mathrm{met}} T_{\mathrm{sky}},
$$

where $r_{\text {met }}$ is the total reflectivity of the snow on the metal plate, which comprises the internal reflectivity of the slab and that of the interface and can be expressed as follows:

$$
r_{\mathrm{met}}=r_{i}+\left(1+r_{i}\right)^{2} R_{\mathrm{met}}
$$

where $R_{\text {met }}$ is a function of internal transmissivity $(t)$ and reflectivity $(r)$ and of the interface reflectivity $\left(r_{i}\right)$ :

$$
R_{\mathrm{met}}=\frac{r+t^{2}(1-r)^{-1}}{1-r r_{i}-r_{i} t^{2}(1-r)^{-1}}
$$


b. Brightness temperature of snow on absorber $\left(T b_{a b s}\right)$. Similar to the case of the snow on metal plate, the upwelling brightness temperature of the snow on absorber $\mathrm{Tb}_{\mathrm{abs}}$ is [Fig. $\underline{4(\mathrm{~b})}$ ]

$$
\mathrm{Tb}_{\mathrm{abs}}=\left(1-r_{\mathrm{abs}}\right) T_{\mathrm{snow}}+r_{\mathrm{abs}} T_{\mathrm{sky}},
$$

where it is assumed that the snow and absorber temperature are the same and that $r_{\mathrm{abs}}$ is the total reflectivity of the snow on the absorber, including the internal reflectivity of the slab $(r)$ and that of the interface air/snow $\left(r_{i}\right)$ :

$$
r_{\mathrm{abs}}=r_{i}+\left(1-r_{i}\right)^{2} R_{\mathrm{abs}},
$$

where $R_{\text {abs }}$ similarly to $R_{\text {met }}$ is expressed as follows:

$$
R_{\mathrm{abs}}=\frac{r+r_{i} t^{2}\left(1-r r_{i}\right)^{-1}}{1-r r_{i}-\left(r_{i} t\right)^{2}\left(1-r r_{i}\right)^{-1}}
$$

The internal reflectivities $(r)$ and transmissivities $(t)$ can be computed from the measured $\mathrm{Tb}_{\text {met }}$ and $\mathrm{Tb}_{\mathrm{abs}}$ if $r_{i}$ (air-snow interface) is known. If the snow interface is considered smooth, $r_{i}$ can be computed using the Fresnel reflectivity formula at the given incidence angle, polarization, and dielectric slab permittivity $\varepsilon$. To obtain the values of $r$ and $t$, we just need to

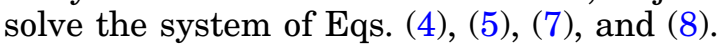

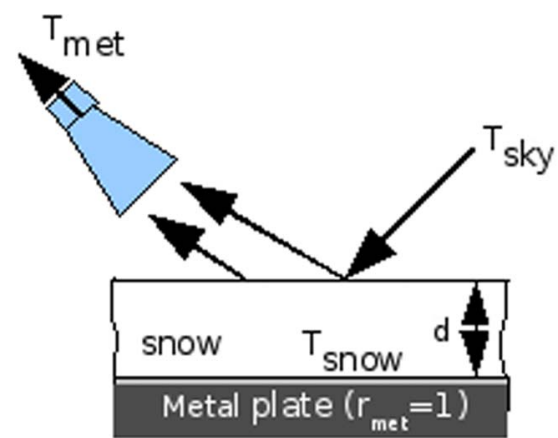

(a)

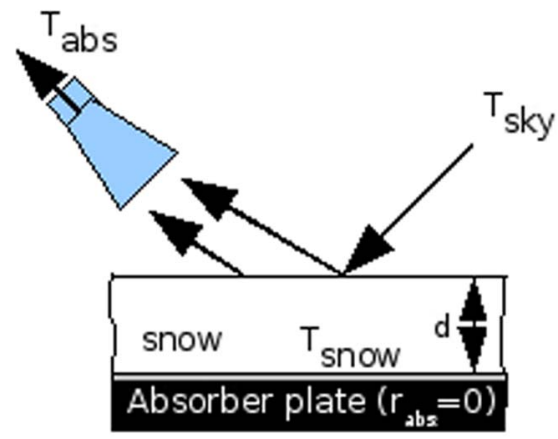

(b)

Fig. 4. (Color online) Principles of snow sample measurements: (a) brightness temperature $\mathrm{Tb}_{\text {met }}$ of snow on metal plate and (b) brightness temperature $\mathrm{Tb}_{\mathrm{abs}}$ of snow on absorber. Corresponding values of blackbody radiation $\mathrm{Tb}_{\mathrm{bb}}$ were also measured.
To link the slab internal reflectivity $r$ and transmissivity $t$ of our samples on the one hand, to the internal scattering and absorption coefficients on the other hand, we used the six-flux model proposed by Wiesmann et al. [24]. The six-flux model is a simplified radiative transfer model that helps to account for all the radiation that is propagating in the snow slab. It is a model that reduces the radiation at a given polarization to six-flux streams along and opposed to the three principal axes of the slab. The horizontal fluxes represent the trapped radiation whose internal incidence angle $\theta$ is greater than the critical angle for total reflection $\theta_{c}$ :

$$
\theta>\theta_{c}=\arcsin \sqrt{1 / \varepsilon}
$$

where $\varepsilon$ is the relative permittivity of the slab.

The vertical fluxes represent the radiation which does not undergo total reflection; this is the radiation that interacts with the space above the snowpack. In the case of plane-parallel and isotropic slabs, the sixflux model is reduced to the well-known two-flux model, and the two-flux absorption coefficient $\gamma^{\prime}{ }_{a}$ and scattering coefficient $\gamma^{\prime}{ }_{b}$ can be written in terms of six-flux parameters:

$$
\gamma_{a}^{\prime}=\gamma_{a}\left(1+4 \gamma_{c}\left(\gamma_{a}+2 \gamma_{c}\right)^{-1}\right)
$$

$$
\gamma_{b}^{\prime}=\gamma_{b}+4 \gamma_{c}^{2}\left(\gamma_{a}+2 \gamma_{c}\right)^{-1},
$$

where $\gamma_{a}$ is the six-flux absorption coefficient, $\gamma_{b}$ is the six-flux backscattering coefficient, and $\gamma_{c}$ is the corner scattering coefficient (the coefficient for coupling between the vertical and the horizontal fluxes). Wiesmann et al. [24] also established that, for a snow layer of thickness $d$, the internal reflectivity $(r)$ and transmissivity $(t)$ can be expressed as

$$
r=r_{0}\left(1-t_{0}^{2}\right)\left(1-r_{0}^{2} t_{0}^{2}\right)^{-1}
$$

$$
t=t_{0}\left(1-r_{0}^{2}\right)\left(1-r_{0}^{2} t_{0}^{2}\right)^{-1},
$$

where the one-way transmissivity $t_{0}$ of the slab is

$$
t_{0}=\exp (-\gamma d /(\cos \theta))
$$

and the reflectivities $r_{0}$ of infinite slab thickness is

$$
r_{0}=\gamma_{b}{ }^{\prime}\left(\gamma_{a}{ }^{\prime}+\gamma_{b}{ }^{\prime}+\gamma\right)^{-1}
$$

and a function of the two-flux absorption, scattering coefficients $\left(\gamma^{\prime}{ }_{a}\right.$ and $\left.\gamma^{\prime}{ }_{b}\right)$, and the damping coefficient $\gamma$, which can also be expressed as a function of $\gamma^{\prime}{ }_{a}$ and $\gamma_{b}^{\prime}$ :

$$
\gamma=\left(\gamma_{a}^{\prime}\left(\gamma_{a}{ }^{\prime}+2 \gamma_{b}{ }^{\prime}\right)\right)^{1 / 2}
$$


For an isotropic scattering, the total scattering coefficient $\gamma_{s}$ is given by the sum

$$
\gamma_{s}=2 \gamma_{b}+4 \gamma_{c}
$$

and the ratio $2 \gamma_{c} / \gamma_{b}$ can be expressed as follows:

$$
2 \gamma_{c} / \gamma_{b}=x /(1-x)
$$

where $x$ is

$$
x=((\varepsilon-1) / \varepsilon)^{1 / 2} .
$$

From Eqs. (10)-(19) we have the full picture of how the internal reflectivity $r$ and transmissivity $t$ are linked to the six-flux scattering coefficients $\gamma_{b}, \gamma_{c}$, and $\gamma_{s}$. We solved the system of Eqs. (10)-(19) to derive the scattering coefficients.

\section{Results and Discussion}

The scatterplot in Fig. 5 shows a trend for SSA to increase with decreasing density. The plot shows characteristic SSA-density clusters for newly fallen snow (density, $\rho<200 \mathrm{~kg} \cdot \mathrm{m}^{-3}$ shown in triangles), rounded grain $\left(200<\rho<300 \mathrm{~kg} \cdot \mathrm{m}^{-3}\right.$ shown in filled circles) and for compacted rounded grain snow $\left(\rho>300 \mathrm{~kg} \cdot \mathrm{m}^{-3}\right.$ shown in diamonds). The same clusters were also found by Matzl and Schneebeli [22], and it is assumed that this loose relationship is due to the fact that metamorphism and sintering often have an impact on both SSA and density at the same time. In fact, Kerbrat et al. [21] have shown that SSA is not correlated to snow density. The loose relation that seems to suggest a correlation between SSA and density is due to a side effect that, on average, when the snowpack increases in density, its SSA decreases. That is not always the case. Rosenfeld and Grody [25] also demonstrated that from midseason,

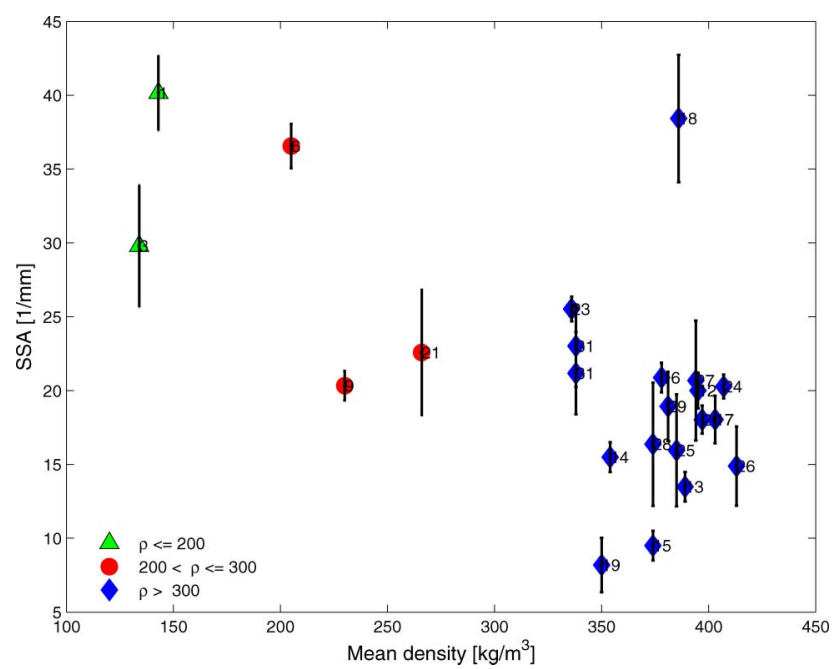

Fig. 5. (Color online) Scatterplot of SSA and snow mean density. Three different types of snow are distinguished: snow with density $\rho<200 \mathrm{~kg} \cdot \mathrm{m}^{-3}$ (shown as triangles), density $200<\rho<300 \mathrm{~kg} \cdot \mathrm{m}^{-3}$ (shown as filled circles), and snow with density $\rho>300 \mathrm{~kg} \cdot \mathrm{m}^{-3}$ (shown as diamonds). an internal restructuring of the snow occurs that can dramatically affect SSA even though the total mass of individual particles, as well as the total density of the snowpack, remain constant.

The representation of the scattering coefficient at 21 and $35 \mathrm{GHz}$ at vertical polarization versus SSA [Figs. 6(a) and 6(b)] shows an exponential decrease of the scattering coefficient with the snow specific surface. The correlation coefficients between the scattering coefficient at 21 and $35 \mathrm{GHz}$ at vertical polarization and the SSA are -0.57 and -0.64 , respectively. New snow with high SSA has a low scattering coefficient, and settled snow with rounded grains has low SSA and a high scattering coefficient. Samples 15 and 19, taken at the bottom of the snowpack and made up of coarse grains, have very low SSA and a very high scattering coefficient. The same trend can be seen at horizontal polarization [Figs. $6(\mathrm{c})$ and $6(\mathrm{~d})$ ]. The correlation coefficients between the scattering coefficient at 21 and $35 \mathrm{GHz}$ at horizontal polarization and the SSA are -0.64 and -0.77 , respectively.

Figure 7 is the representation of the scattering coefficient at 21 and $35 \mathrm{GHz}$ at vertical polarization [Figs. 7(a) and 7(b)] and at 21 and $35 \mathrm{GHz}$ at horizontal polarization [Figs. 7(c) and 7(d)] versus snow density. As expected, there is no correlation between the scattering coefficients and the snow density. Denser samples are not always characterized by larger scattering coefficients. These results show that the scattering is mainly dependent on SSA. Foster et al. [26] also demonstrated that the SSA is so dominant in snow microwave scattering that the cumulative contribution of other structural features is overwhelmed.

According to Mätzler [8] and Debye et al. [12], the relation between the correlation length $(\mathrm{pc})$ and the SSA is as follows:

$$
\mathrm{pc}=4 \frac{(1-v)}{\mathrm{SSA}}
$$

where $v=\rho / \rho_{\text {ice }}$ is the volume fraction of the ice ( $\rho$ is the density of the snow and $\rho_{\text {ice }}=917 \mathrm{~kg} / \mathrm{m}^{3}$ is the density of ice).

We used the formula to convert the SSA derived from the NIR photography into the correlation length. The representations of the pc versus the scattering coefficient at 21 and $35 \mathrm{GHz}$ at vertical polarization [Figs. 8(a) and 8(b)] show increasing scattering coefficient with pc. Coarse snow grains have high correlation lengths, which translate into high scattering coefficients. The same trend can also be seen for the scattering coefficients at 21 and $35 \mathrm{GHz}$ at horizontal polarization [Figs. 8(c) and $8(\mathrm{~d})]$.

Similar results were found by Wiesmann et al. [24] on samples collected during the winters of 1994/1995 and 1995/1996 using the same methodology to extract the scattering coefficient and cold laboratory measurements to determine the pc. The plots of 
(a)

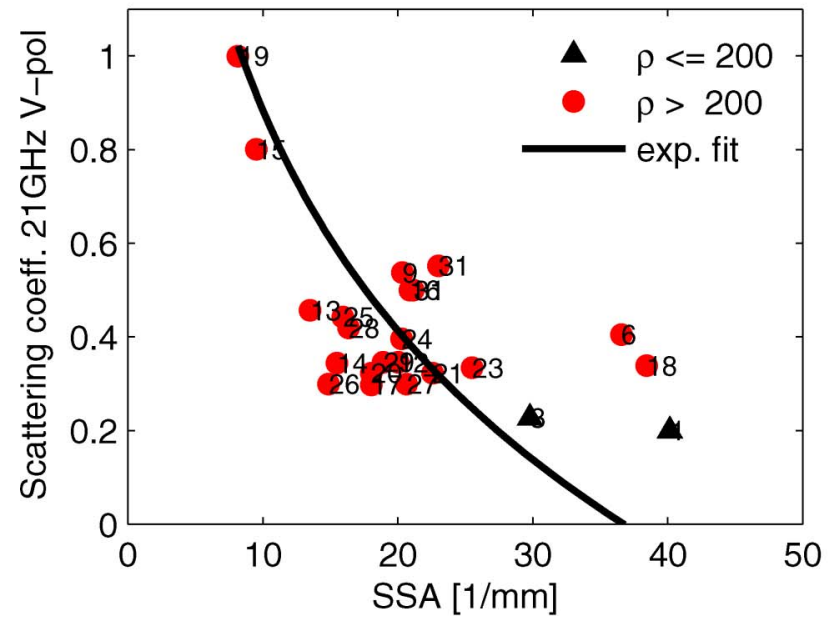

(c)

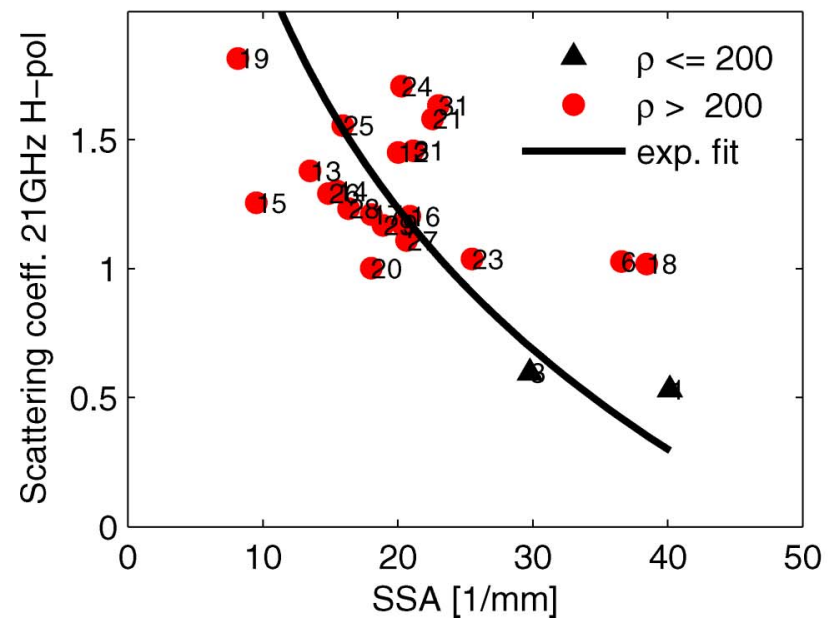

(b)

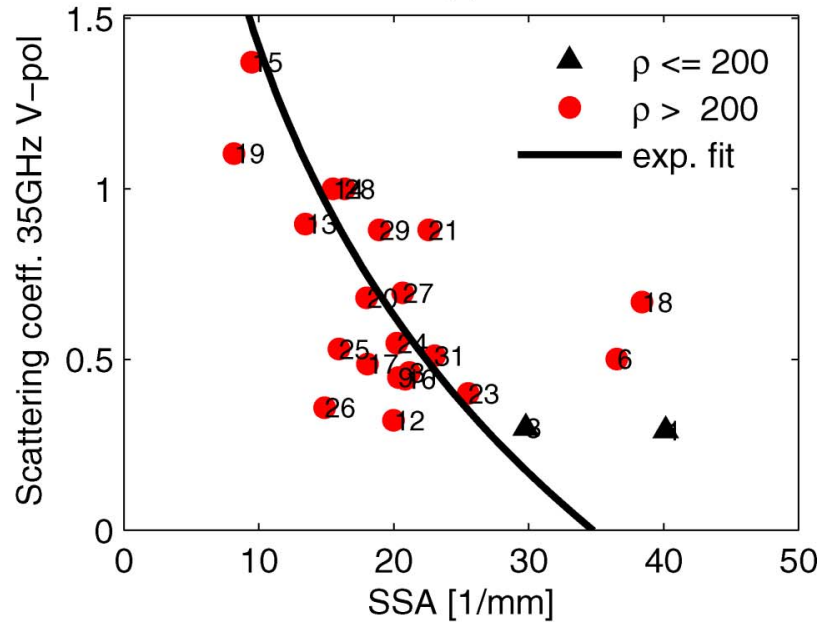

(d)

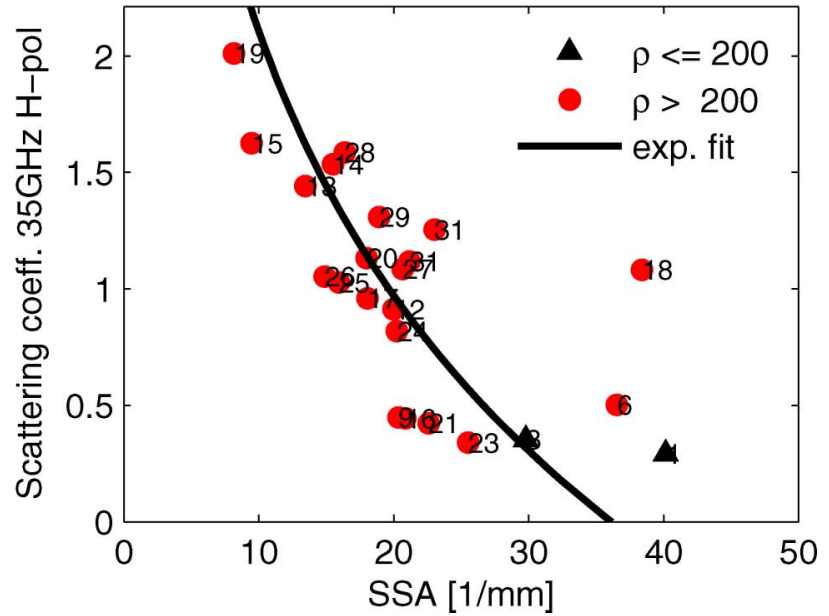

Fig. 6. (Color online) Representation of the scattering coefficient at (a) $21 \mathrm{GHz}$ vertical polarization, (b) $35 \mathrm{GHz}$ vertical polarization, (c) $21 \mathrm{GHz}$ horizontal polarization, and (d) $35 \mathrm{GHz}$ horizontal polarization versus the SSA. Samples with density $\rho<200 \mathrm{~kg} \cdot \mathrm{m}^{-3}$ are shown as triangles. Samples with density $\rho>200 \mathrm{~kg} \cdot \mathrm{m}^{-3}$ are shown as filled circles. The numbers on the right of the symbols indicate the sample's number. The solid curve is the exponential fit of the dense samples.

correlation length versus scattering coefficients at vertical and horizontal polarization of the samples with densities above $200 \mathrm{~kg} \cdot \mathrm{m}^{-3}$ are fitted with a power law fit:

$$
\gamma_{s p}=d_{2 p} \cdot(\mathrm{pc})^{c_{3 p}}
$$

The fits are shown by the solid lines. The parameters $d_{2 p}$ and $c_{3 p}$, ( $p$ represents the vertical or horizontal polarization) and their equivalent found by Wiesmann et al. [24] $\left(d_{2 \text { wie }}, c_{2 \text { wie }}\right)$ are provided in Table 2. The pc we found are in the same range as those of Wiesmann et al. [24], but our scattering coefficients are generally higher than those found by them. The power law fits (Fig. 8) in [24] and are steeper compared to those of our data. The first possible reason for these discrepancies between our data and that of [24], especially at $21 \mathrm{GHz}$ and horizontal polarization could be due to mechanical damage of the radiometer antenna at $21 \mathrm{GHz}$. The antenna tended to shake slightly when a change in polarization from vertical to horizontal was made. The discrepancies could also arise from the fact that there was a difference between our experimental setup and that of [24]. They used a metal frame of the same size as the samples to hold together the snow slabs. Using the frame reduces the edge effects because the frame acts as a mirror for radiation hitting the edge, and thus simulates a larger sample. More importantly, most of our samples were made up of very hard densified snow and the values of pc are mostly concentrated between 0.1 and $0.2 \mathrm{~mm}$. The 2005/2006 winter season was exceptional. The snow depth was about $2 \mathrm{~m}$, while the average snow depth usually recorded in the area is $1.5 \mathrm{~m}[27,28]$. This means that the metamorphism yielded different types of snow grain. For snow density exceeding approximately $350 \mathrm{~kg} \cdot \mathrm{m}^{-3}$, which is the case for most of our samples, 
(a)

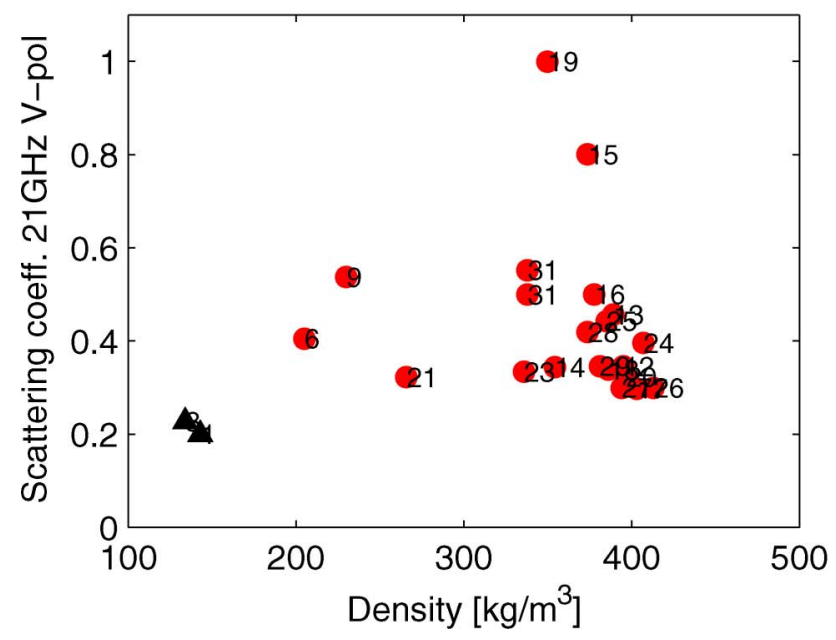

(c)

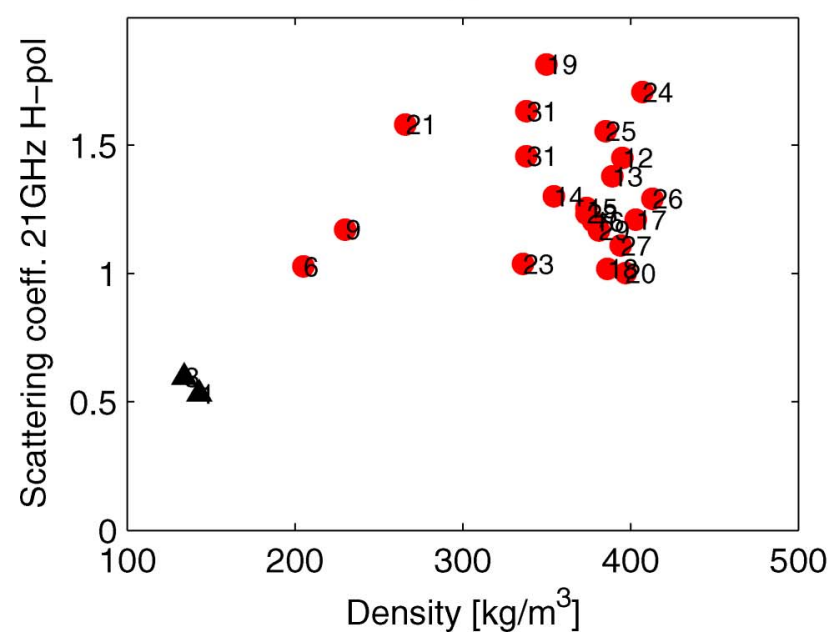

(b)

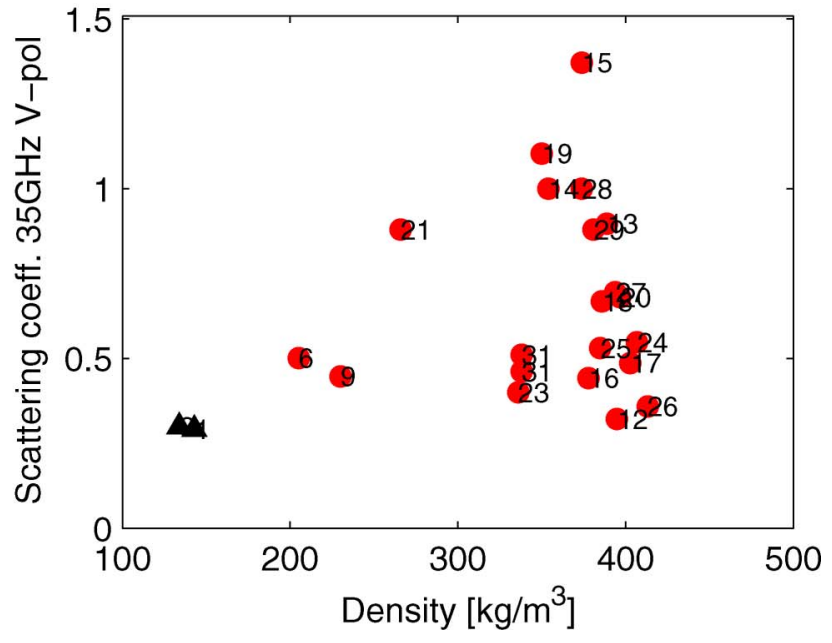

(d)

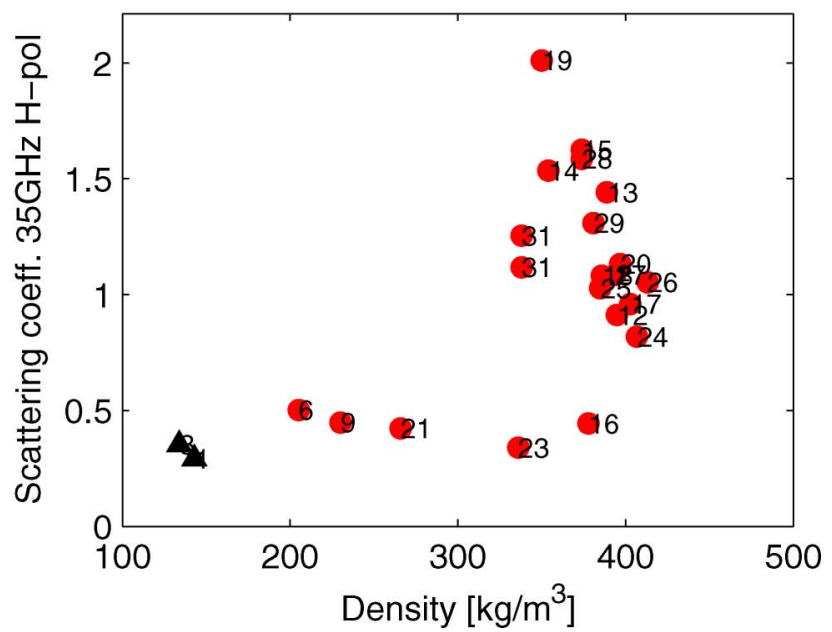

Fig. 7. (Color online) Representation of the scattering coefficient at (a) $21 \mathrm{GHz}$ vertical polarization, (b) $35 \mathrm{GHz}$ vertical polarization, (c) $21 \mathrm{GHz}$ horizontal polarization, and (d) $35 \mathrm{GHz}$ horizontal polarization versus snow density. The numbers on the right of the symbols indicate the sample's number.

pore spaces are very small (due to compaction) and the kinetic growth process is limited. "Hard" depth hoar develops $[29,30]$ under these conditions. Hard depth hoar crystals are composed of sharp angular crystals, but are relatively smaller and stronger (due to higher degree of bonding) than classic depth hoar made up of larger faceted cup shaped grains with thinner walls (smaller pc). These larger hollow crystals tend to grow in low density snow with large pore spaces [29]. The hard depth hoar tends to have a higher pc than the classic one.

The near-infrared reflectance depends on the optical grain size, which is inversely related to SSA but is independent of snow density. As already mentioned at the beginning of this section, SSA and snow density are not correlated. Snow is a random granular medium in which grains cannot be considered as independent scatterers [10]. The snow emission model MEMLS is based on this principle. This is why it uses the correlation length, a parameter that takes into account both the SSA and the snow density $[\underline{8}, \underline{10}, \underline{12}]$.
The estimated correlation lengths from the NIR technique and measured snow parameters, including density, temperature, and sample thickness were used as input parameters in MEMLS to simulate the brightness temperatures at 21 and $35 \mathrm{GHz}$. Figure 9 shows the comparison between the measured brightness temperature of a snow sample placed on a metal plate and snow emission model (MEMLS) predictions. Figures. 9(a) and 9(b) are the results for $21 \mathrm{GHz}$ at vertical and horizontal polarizations. Figures $9(\mathrm{c})$ and $9(\mathrm{~d})$ represent the results for $35 \mathrm{GHz}$ at vertical and hroizontal polarizations. The correlation coefficient ranges from 0.55 at $21 \mathrm{GHz}$ to 0.75 at $35 \mathrm{GHz}$. There can be several reasons for the scatter, especially at $21 \mathrm{GHz}$, including the fact that volume scattering is stronger at $35 \mathrm{GHz}$ than at $21 \mathrm{GHz}$. Furthermore, a systematic error was added to the measurements at $21 \mathrm{GHz}$ because, during the measurements, the $21 \mathrm{GHz}$ radiometer was less stable than the $35 \mathrm{GHz}$ instrument. 
(a)

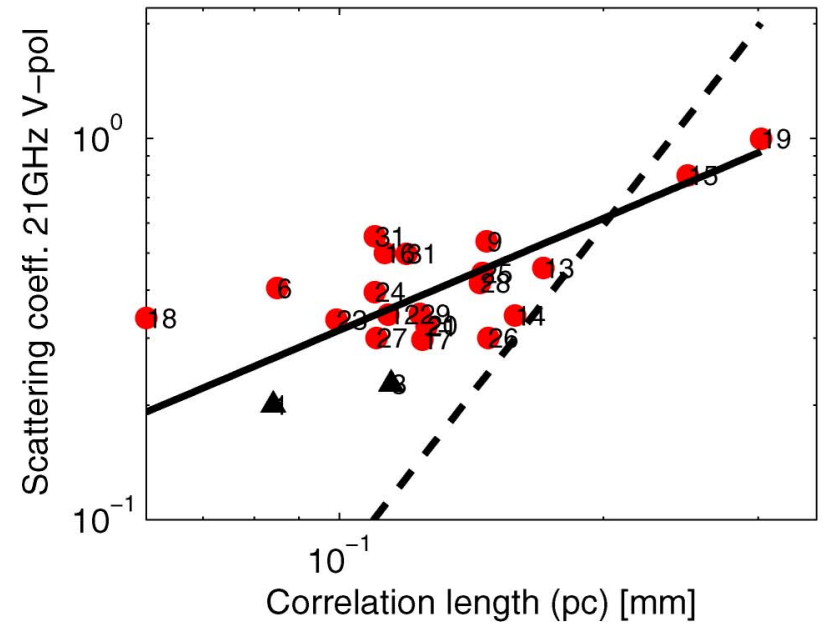

(c)

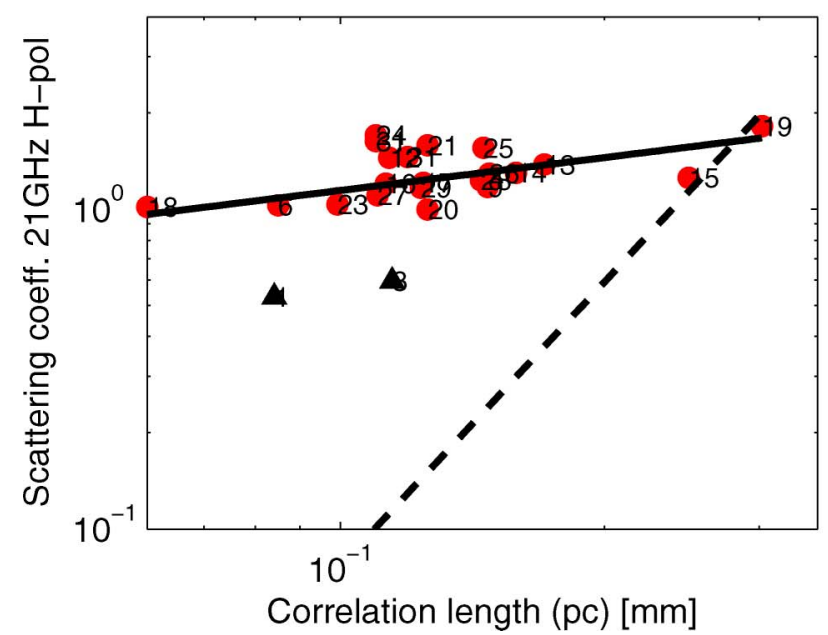

(b)

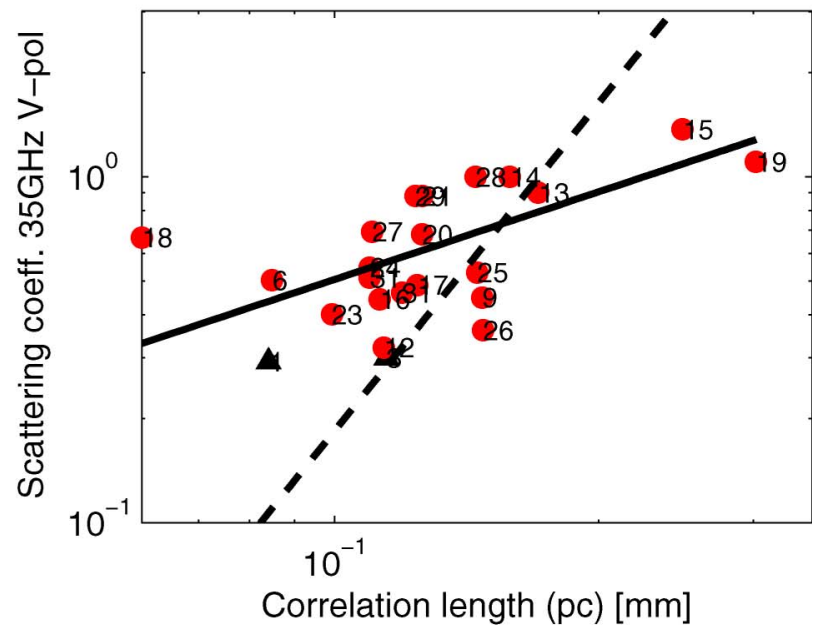

(d)

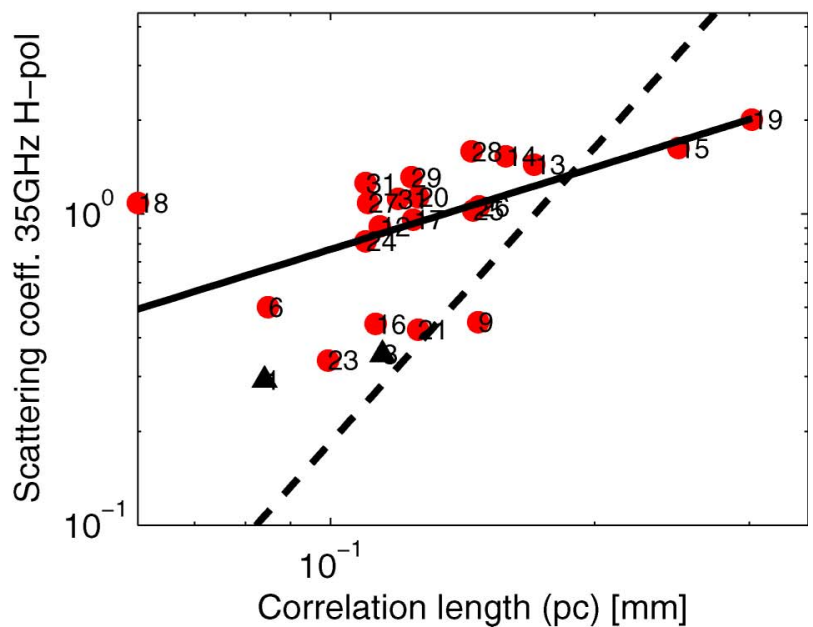

Fig. 8. (Color online) Double logarithmic representation of the scattering coefficients at (a) $21 \mathrm{GHz}$ vertical polarization, (b) $35 \mathrm{GHz}$ vertical polarization, (c) $21 \mathrm{GHz}$ horizontal polarization, and (d) $35 \mathrm{GHz}$ horizontal polarization versus the correlation length. Samples with a density $\rho<200 \mathrm{~kg} \cdot \mathrm{m}^{-3}$ are shown as triangles; samples with a density $\rho>200 \mathrm{~kg} \cdot \mathrm{m}^{-3}$ are shown as filled circles. The numbers on the right of the symbols indicate the sample's number. The solid lines are the power law fit of the dense samples; the broken lines represent the power law fit found by Wiesmann et al. [24].

\section{Conclusion}

We used the relations established by Debye et al. [12] and by Matzl and Schneebeli [22], respectively, to link infrared reflectances of snow to the SSA, then the SSA to the correlation length, knowing the density of snow. From the measurements of brightness temperatures at 21 and $35 \mathrm{GHz}$, we derived the scattering coefficient of the samples by inverting the sandwich and six-flux radiative transfer models. We showed that the scattering coefficient increases with the correlation length. The relationship can be defined by a power law.

The pc we found are in the same range as those determined by Wiesmann et al. [24]. New snow has a pc range from 0.06 to $0.08 \mathrm{~mm}$, settled densified snow is between 0.08 and $0.2 \mathrm{~mm}$, and the depth hoar range is

Table 2. Fit Parameters for the Six-Flux Scattering Coefficient Versus the Correlation Length and the Correlation Coefficients ${ }^{a}$

\begin{tabular}{cccccccc}
\hline Frequency $(\mathrm{GHz})$ & Polarization & $d_{2},\left[\mathrm{~m}^{-1}\right]$ & $c_{3}$ & $R$ & $d_{2 \text { wie }},\left[\mathrm{m}^{-1}\right]$ & $c_{3 \text { wie }}$ & $R_{\text {wie }}$ \\
\hline \multirow{2}{*}{21} & Vertical & 2.967 & 0.975 & 0.83 & 68.3 & 2.95 & 058 \\
& Horizontal & 2.514 & 0.342 & 0.44 & - & - \\
\multirow{2}{*}{35} & Vertical & 3.514 & 0.843 & 0.73 & 260.4 & 3.13 & 0.90 \\
& Horizontal & 5.781 & 0.876 & 0.73 & - & - \\
\hline
\end{tabular}

$\left.{ }^{a} d_{2}, c_{3}, R\right)$ compared to those determined by [24] $\left(d_{2 \text { wie }}, c_{3 \text { wie }}, R_{\text {wie }}\right)$. 

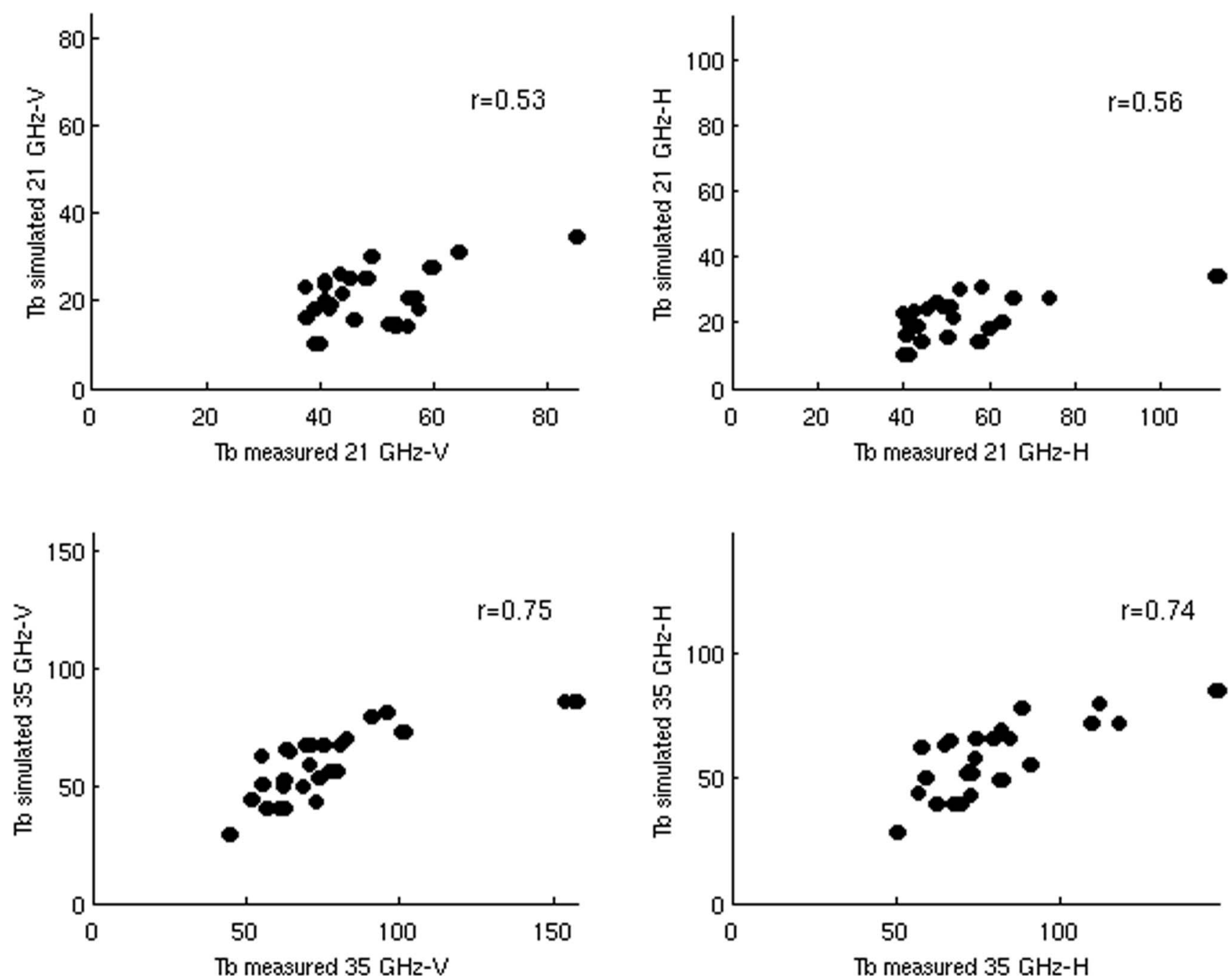

Fig. 9. Comparison of measured Tb of snow placed on a metal plate with snow emission model (MEMLS) predictions: (a) and (b) represent the results at $21 \mathrm{GHz}$ vertical and horizontal polarization; (c) and (d) represent the results at $35 \mathrm{GHz}$ vertical and horizontal polarization.

between 0.2 and $0.4 \mathrm{~mm}$. This confirms the theoretical relationship [Eq. (20)] established by Debye et al. [12] that connects the snow correlation length to its specific surface. The technique shows great potential in the determination of snow correlation length under field conditions, even though it still needs some improvements (i.e., elements such as uniform illumination of the snow-pit wall). The improvement of the determination of the correlation length is critical in the solving of the many-to-one relationship between the snow water equivalent and the measured brightness temperature through the data assimilation scheme.

This work was supported by the National Science and Engineering Research Council of Canada and by Environment Canada (CRYSYS Program). We are grateful to the Institute of Applied Physics (IAP), University of Bern, for the invaluable logistical support they provided us with for the field work, and to the Centre de Calcul Scientifique (CCS) of Université de Sherbrooke and especially to HuiZhong Lu who has been useful in helping to optimize the inversion program.

\section{References}

1. J. T. Pulliainen, J. Grandell, and M. T. Hallikainen, "HUT snow emission model and its applicability to snow water equivalent retrieval," IEEE Trans. Geosci. Remote Sens. 37, 1378-1390 (1999).

2. A. Wiesmann and C. Mätzler, "Microwave emission model of layered snowpacks," Remote Sens. Environ. 70, 307-316(1999).

3. L. Tsang, C.-T. Chen, A. T. C. Chang, J. Guo, and K.-H. Ding, "Dense media radiative transfer theory based on quasicrystalline approximation with application to passive microwave remote sensing of snow," Radio Sci. 35, 731-749 (2000).

4. Y.-Q. Jin, Electromagnetic Scattering Modelling for Quantitative Remote Sensing (World Scientific, 1993).

5. L. Tsang and J. A. Kong, "Application of strong fluctuation random medium theory to scattering from a vegetation-like half space," IEEE Trans. Geosci. Remote Sens. GE-19, 6269 (1981).

6. S. Colbeck, E. Akitaya, R. Armstrong, H. Gubler, J. Lafeuille, K. Lied, D. McClung, and E. Morris, International Classification for Seasonal Snow on the Ground (University of Colorado, 1990).

7. R. L. Armstrong, A. Chang, A. Rango, and E. Josberger, "Snow depths and grain-size relationships with relevance for passive microwaves studies," Ann. Glaciol. 17, 171-176 (1993). 
8. C. Mätzler, "Relation between grain size and correlation length of snow," J. Glaciol. 48, 461-466 (2002).

9. T. C. Grenfell and S. G. Warren, "Representation of a nonspherical ice particle by a collection of independent spheres for scattering and absorption of radiation," J. Geophys. Res. 104, 31697-31709 (1999).

10. C. Mätzler, "Autocorrelation function of granular media with free arrangement of spheres, spherical shells or ellipsoids," J. Appl. Phys. 81, 1509-1517 (1997).

11. R. Parwani, "Correlation function, "http://staff.science.nus .edu.sg/ parwani/c1/node2.html.

12. P. Debye, H. R. Anderson, and H. Brumberger, "Scattering by an inhomogeneous solid II. The correlation function and its applications,” J. Appl. Phys. 28, 679-683 (1957).

13. A. Stogryn, "Correlation functions for random granular media in strong fluctuation theory," IEEE Trans. Geosci. Remote Sens. GE-22, 150-154 (1984).

14. H. Lim, M. E. Veysoglu, S. H. Yueh, R. T. Shin, and J. A. Kong, "Random medium model approach to scattering from a random collection of discrete scatters," J. Electromagn. Waves Appl. 8, 801-817 (1994).

15. J. Giddings and E. Lachapelle, "Diffusion theory applied to radiant energy distribution and albedo of snow," J. Geophys. Res. 66, 181-189 (1961).

16. S. G. Warren and W. J. Wiscombe, "A model for the spectral albedo of snow. I: Pure snow," J. Atmos. Sci. 37, 2734-2745 (1980).

17. J. Dozier, S. R. Schneider, J. McGinnis, and F. Davis, "Effect of grain size and snowpack water equivalent on visible and nearinfrared," Water Resour. Res. 17, 1213-1221 (1981).

18. A. Nolin and J. Dozier, "A hyperspectral method for remotely sensing the grain size of snow," Remote Sens. Environ. 74, 207-216 (2000).

19. D. L. Mitchell, "Effective diameter in radiative transfer: general definition, application, and limitation," J. Atmos. Sci. 59, 2330-2346 (2002).
20. L. Legagneux, A. Cabanes, and F. Dominé, "Measurement of the specific surface area of 176 snow samples using methane adsorption at 77 K," J. Geophys. Res. 107, 4335 (2002).

21. M. Kerbrat, B. Pinzer, T. Huthwelker, H. W. Gäggeler, M. Ammann, and M. Schneebeli, "Measuring the specific surface area of snow with x-ray tomography and gas adsorption: comparison and implications for surface smoothness," Atmos. Chem. Phys. 8, 1261-1275 (2008).

22. M. Matzl and M. Schneebeli, "Measuring specific surface area of snow by near-infrared photography," J. Glaciol. 52, 558564 (2006).

23. T. H. Painter, N. P. Molotch, M. Cassidy, M. Flanner, and K. Steffen, "Contact spectroscopy for determination of stratigraphy of snow optical grain size," J. Glaciol. 53, 121127 (2007).

24. A. Wiesmann, C. Mätzler, and T. Wiese, "Radiometric and structural measurements of snow samples," Radio Sci. 33, 273-289 (1998).

25. S. Rosenfeld and N. C. Grody, "Metamorphic signature of snow revealed in SSM/I measurements," IEEE Trans. Geosci. Remote Sens. 38, 53-63 (2000).

26. J. L. Foster, D. K. Hall, A. T. C. Chang, A. Rango, W. Wergin, and E. Erbe, "Effects of snow crystal shape on the scattering of passive microwave radiation," IEEE Trans. Geosci. Remote Sens. 37, 1165-1168 (1999).

27. T. Weise, "Radiometric and structural measurements of snow," PhD thesis (Inst. of Appl. Physics University of Bern, 1996).

28. M. Laternser and M. Schneebeli, "Long-term snow climate trends of the Swiss Alps (1931-99)," Int. J. Climatol. 23, 733-750 (2003).

29. E. Akitaya, "Studies on depth hoar," Contrib. Inst. Low Temp. Sci. Hokkaido Univ. Ser. A 26, 1-67 (1974).

30. D. Marbouty, "An experimental study of temperature-gradient metamorphism,” J. Glaciol. 26, 303-312 (1980). 